\title{
Central laboratory and point of care assessment of perioperative hemostasis
}

\author{
[Examens de l'hémostase périopératoire réalisés en laboratoire et au lit du patient]
}

Pierre Sié MD PHD, ${ }^{*}$ Annick Steib MD ${ }^{\dagger}$

Purpose: To review laboratory investigations required for the diagnosis of a constitutional or acquired defect of hemostasis before surgery, or during the perioperative period in context of rapid evolution and possible therapeutic adjustment.

Methods: A review of the literature.

Principal findings: Systematic preoperative screening is poorly efficient. It should be restricted to patients selected on clinical history and physical examination. Intra- and postoperative investigation is oriented by the clinical circumstances. In most instances, laboratory investigation is organized as hierarchical steps, the first one including a platelet count, a prothrombin time and activated partial thromboplastin time. According to the results of these assays and to the clinical circumstances, further tests specific of primary hemostasis, coagulation or fibrinolysis are performed. Point of care (POC) monitoring has been developed more recently for the investigation of a perioperative bleeding. Several tests examine platelet functions, others measure whole blood activated partial thromboplastin time or prothrombin time and some explore global hemostasis, allowing the detection of excessive fibrinolysis. Point of care testing provides a rapid and valuable answer but, if one accepts the monitoring of unfractionated heparin during extracorporeal circulation, most assays have not been properly validated.

Conclusion: The investigation of hemostasis at the central laboratory and POC testing have distinct objectives. The utility of the former for the diagnosis and the adjustment of therapeutics have been well demonstrated. In contrast, the experience with POC testing is relatively recent, and its utility for patient management remains to be demonstrated in proper clinical trials.
Objectif : Proposer une revue des examens de laboratoire d'hémostase et des tests "au lit du patient» utilisés pour la surveillance et la prise en charge thérapeutique en période périopératoire.

Méthode : Revue de la littérature.

Principaux résultats : L'exploration de l'hémostase au laboratoire central est orientée vers le diagnostic d'anomalies constitutionnelles ou acquises avant la chirurgie ou en période périopératoire. Elle participe à la surveillance d'anomalies acquises qui peuvent être rapidement évolutives et à l'ajustement des thérapeutiques correctrices. L'exploration systématique par des tests préopératoires est peu efficace et doit être abandonnée. Les patients ayant des antécédents et un examen clinique pouvant faire soupçonner un trouble de l'hémostase doivent seuls être explorés. Dans la plupart des cas, cette exploration est organisée en étapes successives de complexité croissante. La première étape inclut une numération plaquettaire, un temps de Quick (taux de prothrombine) et un temps de céphaline avec activateur. En fonction des résultats de cette première étape et du contexte clinique, des tests plus spécifiques de l'hémostase primaire, de la coagulation ou de la fibrinolyse sont exécutés secondairement. L'exploration au lit du malade ou au bloc opératoire est particulièrement utile dans les situations de saignement périopératoire. II existe plusieurs appareils permettant de tester les fonctions des plaquettes, de mesurer le taux de prothrombine et le temps de céphaline avec activateur sur sang total, ou d'enregistrer le déroulement global du processus de coagulation et de fibrinolyse qui lui succède. De tels systèmes ont l'avantage de donner une réponse rapide, écourtée des délais de transport et de centrifugation des échantillons, mais si l'on fait exception de la surveillance de l'anticoagulation au cours des circulations extra-corporelles, l'ajustement thérapeutique à l'aide de ces tests est rapporté par un petit nombre d'équipes et demande à être validé par des essais cliniques plus larges.

Conclusion : L'exploration de l'hémostase au laboratoire central et au lit du patient ont des objectifs différents. L'utilité des tests de laboratoire pour le diagnostic et l'ajustement thérapeutique est largement démontrée. L'expérience des tests au lit du patient et de leur utilisation pour la prise en charge de celui-ci est encore réduite et reste du domaine de l'investigation clinique.

From the Hematology Laboratory, ${ }^{*}$ Hôpital Purpan, CHU de Toulouse, Toulouse; and the Department of Anesthesiology, ${ }^{\dagger}$ Hôpital Civil, HUS Strasbourg, Strasbourg, France.

Address correspondence to: Dr. Pierre Sié, Hematology Laboratory, Hôpital Purpan, CHU de Toulouse 31059 France.

Phone: 33(0)561779065; Fax: 33(0)561497610; E-mail: iie.p@chu-toulouse.fi 


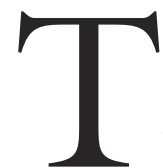

$\mathrm{HE}$ assessment of hemostasis in the perioperative period, a major concern in clinical practice, has multiple objectives: to screen for constitutional or acquired hemorrhagic disorders preoperatively, to recognize compromised hemostasis in bleeding patients intra- and postoperatively, and to monitor the treatment in these circumstances. Investigation at the central laboratory and point of care (POC) testing, which avoids the delay of sample transmission, are complementary approaches. The preoperative investigation, when clinically justified, may involve sophisticated tests, whereas the need for urgent results in actively bleeding patients favours rapid testing and POC methods. The aim of this article is to review, globally, the laboratory assessment of hemostasis, the currently available POC instruments, and the respective place of these approaches in the patient's management.

\section{Laboratory investigation}

\section{Global objectives and process}

Laboratory investigations are oriented exclusively towards the assessment of the bleeding risk. The thrombotic risk is based on clinical information, and the exploration of thrombophilia. Apart from the case of special patient monitoring, e.g., antithrombotic therapy or dose-adjustment during treatment of hemophilia or related disorders, which may directly require specific tests, the laboratory investigation of hemostasis proceeds by successive steps.

The first step includes a limited number of assays which are simple, automated and inexpensive, and able to identify those subjects with a good probability of having no hemostatic defect. The sensitivity of this step is reasonably high, but not enough to exclude all possible defects, so that, in case of clinical suspicion, it should be completed as indicated below. In contrast, its specificity is low and several studies have reported the inefficiency of systematic laboratory screening of preoperative abnormalities known to have a significant impact on the hemorrhagic risk. ${ }^{1-3}$ This is the reason why preoperative laboratory investigations should be restricted to patients with a proven or suspected bleeding history, a possible acquired defect due to the current pathology, or an unreliable history. This first step includes the measurement of the platelet count, activated partial thromboplastin time (aPTT), and prothrombin time (PT).

The second step of investigation is directed by the results of the first one and/or by clinical circumstances. If a defect of primary hemostasis is suspected, the bleeding time or an in vitro equivalent will be performed, followed by platelet function tests, mainly optical aggregometry, and measurement of von Willebrand factor. If coagulation seems to be involved, measurement of clottable fibrinogen, thrombin time, and individual factors of the intrinsic or extrinsic pathways will be performed. If an excessive fibrinolysis is suspected, the measurement of fibrin(ogen) degradation products, mainly D-Dimers, and a global test such as the euglobulin lysis time, will be useful. At the end of this second step, the defect in hemostasis, or the dominant physiopathological mechanism responsible for bleeding will have been identified with sufficient precision to take the appropriate corrective measures. These tests should be relatively simple in order to be repeated as required for patient monitoring.

\section{Preanalytical variables}

The quality of sampling is of paramount importance for the assessment of hemostasis. ${ }^{4}$ Except for the platelet count, performed on EDTA-anticoagulated blood, all tests are performed on platelet-poor plasma from citrated blood. As the anticoagulant used (sodium citrate, 0.105 or $0.129 \mathrm{M}$ ) is liquid, its final concentration in plasma will depend on the anticoagulant:blood ratio, which should be 1:9, and on the hematocrit level, which may vary largely perioperatively. Arterial or venous blood are equally suitable, but caution should be taken to avoid any dilution or contamination of the sample by plasma substitutes or heparin. Difficult, laborious sampling generates traces of thrombin, which activate hemostasis in vitro and yields a false thrombocytopenia, because of platelet aggregates, a decreased fibrinogen concentration, a rise in soluble complexes, and variable changes in aPTT, PT and factor activity, mimicking a consumption coagulopathy. Finally, transport and processing of the sample should be performed within two hours.

\section{Global tests of primary hemostasis}

The template bleeding time suffers from several limitations. It is operator-dependent, poorly reproducible, invasive, and cannot be repeated easily. It is not sufficiently sensitive to detect mild bleeding disorders. Fifty percent of patients with type I von Willebrand disease $(v W D)^{5}$ and about $33 \%$ of patients treated by multiple doses of aspirin ${ }^{6}$ do not display a significant prolongation of bleeding time above baseline. The test is not predictive of surgical bleeding. ${ }^{7-9}$ So, its value as a screening preoperative test is uncertain and its use should be restricted to second line investigation of patients suspected of a disorder of primary hemostasis.

Recently, a simple system, which can also be performed as POC testing, ${ }^{10}$ has been developed to 
mimic in vitro template bleeding time. The Platelet Function Analyser (PFA 100®, Dade-Behring, Marburg, Germany) measures the closure time (time to cessation of flow) of citrated blood aspirated at a high shear rate through the central aperture of a nitrocellulose membrane. Platelets are activated both by the shear stress and by a combination of agonists coated on the membrane, either collagen + epinephrine cartridge or collagen + adenosine diphosphate cartridge. Platelet aggregate formation, responsible for cessation of flow, depends on platelet number and function, and on functional von Willebrand factor. Aspirin usually prolongs the closure time with the collagen + epinephrine, but not with the collagen + adenosine diphosphate cartridge. In contrast, the test is poorly sensitive to thienopyridines. ${ }^{10}$ The test is simple and reproducible (coefficient of variation close to $10 \%$ ), but it is sensitive to preanalytical conditions such as the delay after sampling, sodium citrate concentration or the hematocrit level. It has been evaluated by several groups and seems reasonably sensitive to vWD and to a majority of, but not all, constitutional thrombopathies. ${ }^{10,11}$ Its main advantage is that the results can be obtained rapidly and with far less laboratory work-up, in comparison with standard assays for the diagnosis of vWD or thrombopathies. Its place as a preoperative screening test, for the rapid exclusion of a bleeding diathesis, warrants further investigation.

\section{Specific tests of primary hemostasis}

The platelet count is mandatory as a first line preoperative test and is frequently repeated postoperatively, as thrombocytopenia is frequent, due to consumption, blood loss, sepsis, hemophagocytosis, immunization, or drug reaction, including heparin-induced thrombocytopenia. Errors in the automated platelet count are common, usually in the direction of under-estimation by formation of in vitro platelet microagregates, secondary to poor quality of sampling or, rarely, EDTA-induced agglutination. Recent thrombocytopenia should therefore be controlled by microscopy, and, if necessary, on a citrated blood sample.

Optical platelet aggregometry is the reference assay for diagnosis of platelet disorders, despite not being standardized. ${ }^{12}$ Indeed, the anticoagulant, the platelet agonists, their concentrations and the type of agregometer influence the performance of the test, as does the choice of the endpoints (rate of aggregation, lag-phase, maximum transmittance). The results are modified by platelet counts and in vitro normalization of this variable is not always easy. The laboratory workup is complex and the equipment costly. Thus, the test is restricted to the second line investigation of disor- ders of platelet function, usually associated to platelet membrane antigen analysis by flow cytometry.

The diagnosis of vWD and its variants, when suspected, requires the measurement of von Willebrand factor activity by a ristocetin cofactor assay and of von Willebrand factor antigen. ${ }^{5}$ Rapid automated assay systems are now available and the results, completed by the measurement of factor VIII:c activity, can be obtained within one hour in most laboratories, which may be useful for monitoring desmopressin or substitution therapy of vWD patients.

\section{Prothrombin time and aPTT as screening coagula- tion tests}

The combination of PT and aPTT is the first step of coagulation assessment (Figure 1). The PT and aPTT measure the delay in thrombin threshold generation, via the so-called "extrinsic" and "intrinsic" pathways respectively. They provide little or no information on quantitative thrombin generation, fibrin polymerization, or clot stability. Therefore, mild deficits of fibrinogen, dysfibrinogenemia, and rare but severe bleeding disorders such as complete deficiencies in factor XIII or plasmin/plasminogen activator inhibitors, will be not recognized.

The PT is initiated by the addition of thromboplastin, a mixture of tissue factor, phospholipids and calcium. Results are expressed in seconds $(n=10-14$ $\mathrm{sec}$, according to the reagent), ratio of normal to control $(n \leq 1.3)$, or percent of normal value, arbitrarily $100 \%(n=70-100 \%)$. Due to significant differences between the sensitivity of commercial thromboplastin reagents to the effect of vitamin $\mathrm{K}$ antagonists, the PT of patients treated by oral anticoagulants are expressed as the international normalized ratio (INR), which provides a better standardization $(n=1.0-1.3$ in untreated patients, usual therapeutic range INR = $2-3)$. The PT is sensitive to factors VII, X, V, II, with noticeable differences in responsiveness between the thromboplastins, so that a mild deficiency in one of the above factors may go unnoticed. However, the test is appropriate for the diagnosis of many disorders with clinical significance: severe isolated factor deficiency and combined defects due to hepatic failure, vitamin $\mathrm{K}$ deficiency, dilutional or consumption coagulopathy. A fibrinogen concentration below $0.5 \mathrm{~g} \cdot \mathrm{L}^{-1}$, and thrombin inhibitors such as heparin, hirudin or synthetic peptides at the upper edge of the therapeutic range and above, also prolong the PT.

The aPTT is initiated by the addition of an activator of the contact system, followed by phospholipids and calcium. Results are expressed in seconds $(n=25-40$ $\mathrm{sec}$, according to the analytical system) or in ratio of 

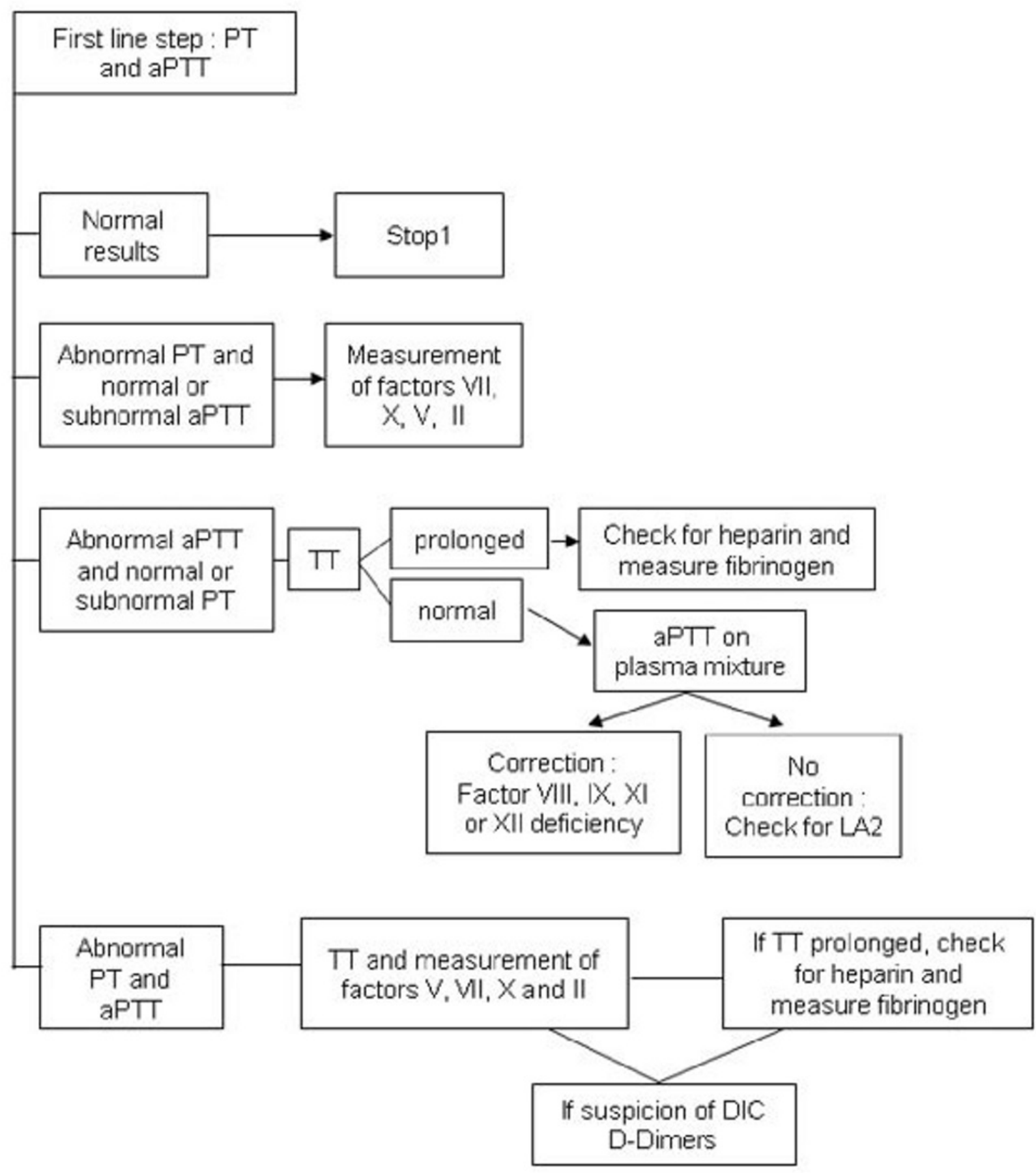

FIGURE 1 Simplified chart of assessment of coagulation at the central laboratory. The first step (screening) usually associates PT and aPTT, giving four possible combinations of results. According to each combination, further assays (second line of investigation) are performed. 1) Specific assays are required for exclusion of rare disorders (see text for details), and fibrinogen measurement if lowered fibrinogen concentrations or dysfibrinogenemia are suspected. 2) The presence of lupus antibodies does not exclude a factor deficiency. Proceed to measure factors VIII, IX and XI. PT = prothrombin time; aPTT $=$ activated partial thromboplastin time. 
normal to control $(n \leq 1.2)$. The aPTT is sensitive to contact factors (high molecular weight kininogen and prekallikrein) and to factors XII, XI, IX, VIII, X, V, II, with variable responsiveness between reagents. A fibrinogen concentration below $1 \mathrm{~g} \cdot \mathrm{L}^{-1}$ moderately prolongs the aPTT. The aPTT is currently used for the dose-adjustment of thrombin inhibitors (with therapeutic ranges of the aPTT ratio ranging from 2 to 3 , but again depending on the reagent). Similarly, most lupus anticoagulants prolong the aPTT. For the purpose of preoperative screening, the use a reagent preferably responsive to factor deficiency (especially factors VIII, IX and XI, in order to pick up mild hemophilia and vWD) is recommended, rather than a reagent responsive to thrombin inhibitors or lupus anticoagulant. The presence of a lupus anticoagulant is easily recognized by failure of aPTT correction on plasma mixing study and confirmed by specific phospholipid-dependent assays. ${ }^{13}$ However, for preoperative testing, exclusion of a factor deficiency by individual factor measurement (see below) becomes mandatory.

According to the results of screening tests, the investigation will stop, if the results of both tests are normal, or continue, as depicted in Figure 1.

\section{Further assessment of a coagulation defect}

Through a limited number of simple assays, immediately available on the remaining plasma, the second line of investigation provides a rapid diagnosis of the coagulation disorder, with sufficient precision for a medical decision to be made.

Because the PT and aPTT are poorly sensitive to disorders of fibrin formation, determination of thrombin time and clottable fibrinogen are the first complementary measures. Individual factor quantification is performed using PT- or aPTT-derived assays, with single deficient factor plasmas and appropriate calibration curves for each factor. Although all clotting factors can be measured using this principle, only a few, combined to the determination of fibrinogen level and platelet count, will suffice. For example, factor V level alone is a good index of liver function. Indeed, the changes observed at timed intervals or following corrective therapy are more informative than a single, albeit complete determination of factor levels.

Exploration of fibrinolysis in current practice is very limited. In case of suspicion of disseminated intravascular coagulation, fibrin(ogen) degradation products, usually D-Dimers are useful. True quantitative assays, with a large working range and good reproducibility at the high levels expected postoperatively, are preferable to semi-quantitative assays. ${ }^{14}$ Acute fibrinolysis can be assessed by the euglobulin lysis time, which is shortened to $<90 \mathrm{~min}(n>180 \mathrm{~min})$, but this test is cumbersome, poorly reproducible, and the response is delayed. A low fibrinogen recovery and short halflife after substitution are the best indicators of excessive consumption. When the differential diagnosis between primary and secondary fibrinolysis is difficult, additional measurements of antithrombin (expected low in disseminated intravascular coagulation and secondary fibrinolysis) and of antiplasmin (low in primary fibrinolysis) are valuable.

\section{Monitoring therapy of specific hemostatic conditions} Substitutive therapy of hemophilia and related disorders requires close dose adjustment by individual factor measurement and systematic assessment of the occurrence of a neutralizing iso-antibody. Monitoring antithrombotic therapy is rarely necessary. Unfractionated heparin, if given at doses $>200 \mathrm{U} \cdot \mathrm{day}^{-1}$, is adjusted on the results of the aPTT. Oral anticoagulant treatment should be monitored by measurement of the INR. The accumulation of low molecular weight heparins in high risk patients is prevented by measurement of peak anti-factor-Xa activity on days two or three after initiation of treatment. ${ }^{15}$ Platelet count assessments three times per week during unfractionated heparin therapy are recommended, whereas such a monitoring is of uncertain importance when low molecular weight heparins are used.

\section{Preoperative evaluation}

Due to their lack of sensitivity and specificity in the prediction of bleeding and the detection of rare coagulation defects and also their high cost, routine, systematic tests of hemostasis cannot be recommended preoperatively in the absence of a significant history or clinical signs of bleeding or thrombosis. In all cases, a detailed questionnaire (Table) should be completed by patients in order to detect those who would benefit from laboratory screening. Rapaport ${ }^{16}$ in 1983 proposed four distinct levels of hematological care, which are still relevant:

- level 1: negative screening history, minor surgery: no testing required;

- level 2: negative screening history, major surgery: obtain platelet count, aPTT;

- level 3: bleeding history suspicious, surgery impairing hemostasis or high risk surgery: obtain platelet count, PT, aPTT, bleeding time or equivalent, fibrinogen level;

- level 4: positive bleeding history: specific tests. 
TABLE Simple questionnaire for screening of disorders of hemostasis ${ }^{16}$

Have you ever bled for a long time or developed a swollen tongue
or mouth after cutting or biting your tongue, cheek or lip?
Do you develop bruises larger than a "silver dollar" without being
able to remember when or how you injured yourself? If so, how
big was the largest of these bruises?
How many times have you had teeth pulled and what was the
longest time that you bled after an extraction? Has bleeding ever
started again the day after extraction?
What operations have you had, including minor surgery such as
skin biopsies? Was bleeding after surgery ever hard to stop? Have
you ever developed unusual bruising in the skin around an area of
surgery or injury?
Have you had a medical problem within the past five years requir-
ing a doctor's care? If so, what was its nature?
What medications including aspirin or any other remedies for
headaches, cold, menstrual cramps or other pains have you taken
within the past seven to nine days?
Has any blood relative had a problem with unusual bruising or
bleeding after surgery? Were blood transfusions required to con-
trol this bleeding?

\section{Point of care monitoring}

Point of care testing of hemostasis has increased since the early 1990s. Bedside monitoring is considered useful by clinicians, especially in emergency situations or in perioperative bleeding as turnaround time for conventional basic testing in central laboratories may often be longer than one hour. Point of care devices are generally easy to use and provide a rapid answer. However, most are not recognized as valid and accurate tools for the assessment of hemostasis by hematologists. Further studies will be required to validate these instruments.

\section{1) Platelet function testing}

Platelet dysfunction may be a major cause of perioperative bleeding. The platelet activated clotting time (ACT) Hemostatus (Medtronic Inc, Parker, CO, USA) measures the ACT without platelet activator in comparison with the ACT obtained with increasing concentrations of added platelet activator. The percentage reduction of the ACT caused by the addition of platelet activator is related to the ability of platelets to be activated and to shorten the coagulation time. ${ }^{17}$ The Hemostatus has been shown to be useful to identify patients requiring desmopressin after cardiopulmonary bypass. ${ }^{18}$ However, correlations with postoperative bleeding are contradictory. The prediction of increased chest drainage was documented in one study $^{18}$ and not demonstrated in others. ${ }^{19,20}$
The Platelet Function Analyser (PFA 100; Dade Behring, Liederbach, Germany) explores the platelet adhesive capacity, as described in the previous section. The Platelet Function Analyser measures the closure time which is increased in acquired and congenital platelet defects, drug-induced platelet dysfunction and vWD. ${ }^{10}$ It may also be modified by the hemoglobin concentration and leukocyte count. Correlations between closure time and perioperative bleeding are significant in some studies ${ }^{21}$ and have not been demonstrated in others. ${ }^{20,22}$ Further clinical investigations are required in this area.

\section{2) Activated coagulation time and heparin monitoring}

The devices used to monitor heparin anticoagulation are described elsewhere. Nowadays, the use of these devices is also recommended for invasive procedures including neuroradiological interventions and percutaneous transluminal coronary angioplasty requiring heparinization.

3) Point of care monitoring of the aPTT, PT and INR The CoaguChek pro DM (Roche diagnostics, Mannheim, Germany) is available for bedside measurement of the aPTT, PT or INR. This laser photometer uses different cartridges. Prothrombin time cartridges contain a thromboplastin reagent calibrated against a reference thromboplastin, thus allowing the expression of the results as the INR for patients treated by oral anticoagulants. Once the cartridge has been inserted into the device, it is warmed to $37^{\circ} \mathrm{C}$ in $60 \mathrm{sec}$. Subsequently, a drop of whole blood $(45 \mu \mathrm{L})$ is applied to the disposable plastic reagent cartridge and the blood is drawn into the reaction chamber by capillary action. Results are obtained in three to six minutes.

Some differences have been reported between results of conventional laboratory testing and the CoaguChek. ${ }^{23}$ Accuracy seems to be better for INR values below 4. Above this limit, the CoaguChek underestimates the INR. ${ }^{24}$ Conflicting results have been published concerning PT and aPTT results. Agreement with laboratory tests was better for the PT compared to the aPTT. ${ }^{23}$ The device underestimates low values of aPTT in patients taking heparin, warfarin or pentasaccharide, and overestimates high values of aPTT with these medications. ${ }^{25}$ In one study, the CoaguChek proved to be helpful by guiding blood component therapy in cardiac surgery and limiting the quantity of transfused fresh frozen plasma and platelets. ${ }^{26}$ Other studies would be useful to determine the place of this POC monitor in the perioperative period. 


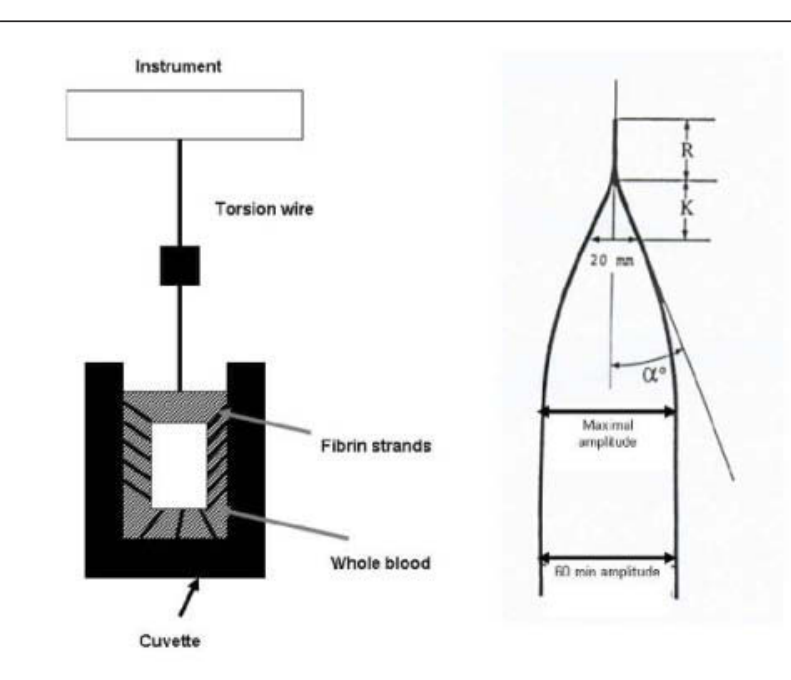

FIGURE 2 Thrombelastography (TEG) variables. $r=$ reaction time (from introduction of the sample until the TEG tracing reaches an amplitude of $2 \mathrm{~mm}$; normal range: six to eight minutes i.e., $12-16 \mathrm{~mm}) ; \mathrm{k}=$ clot formation time, measured from time $\mathrm{r}$ to the point where the amplitude reaches $20 \mathrm{~mm}$ (normal range: three to six minutes i.e., 6-12 mm); $\mathrm{r}+\mathrm{k}=$ coagulation time (normal range: nine to $14 \mathrm{~min}$ i.e., $18-28 \mathrm{~mm}$ ). It is correlated with fibrinogen and platelet concentrations. Alpha angle $=$ angle formed by the slope of the TEG plot from the $r$ to the $k$ value. It gives an indication of the speed at which solid clot forms (normal range: $50-60^{\circ}$ ); Maximum amplitude $(\mathrm{MA})=$ greatest amplitude on the TEG trace; it reflects the absolute strength of the fibrin clot (normal range: $50-70 \mathrm{~mm}$ ). It depends on fibrin and platelets. Clot lysis index $(\mathrm{CLI})=$ $\mathrm{MA} 60 / \mathrm{MA} \times 100$ measures the amplitude at $60 \mathrm{~min}$ following MA and reflects the loss of clot integrity due to lysis (normal range: $85-100 \%$ ). An increase in $\mathrm{r}+\mathrm{k}$ results from hypocoagulability due to defects in coagulation factors, heparin or vitamin $\mathrm{K}$ antagonists. A shortening of $\mathrm{r}+$ $\mathrm{k}$ associated with an increase in MA and alpha angle reflects hypercoagulability. A decrease in CLI indicates hyperfibrinolysis. Hypothermia has no effect on MA.

\section{4) Global evaluation of coagulation}

Two devices allow a global evaluation of coagulation through the exploration of the viscoelastic properties of native blood.

Thrombelastography (TEG; Haemoscope corporation, Stoke, IL, USA) provides a global POC analysis of whole blood coagulation and fibrinolysis. ${ }^{23,27}$ This test assesses the changes in clot strength over time as a small sample of whole blood $(0.35 \mathrm{~mL})$ coagulates in a heated cuvette in which a pin is suspended from a torsion wire. When the clot starts to form, an electric signal is generated from the oscillating cuvette and amplified to produce a typical trace (Figure 2).

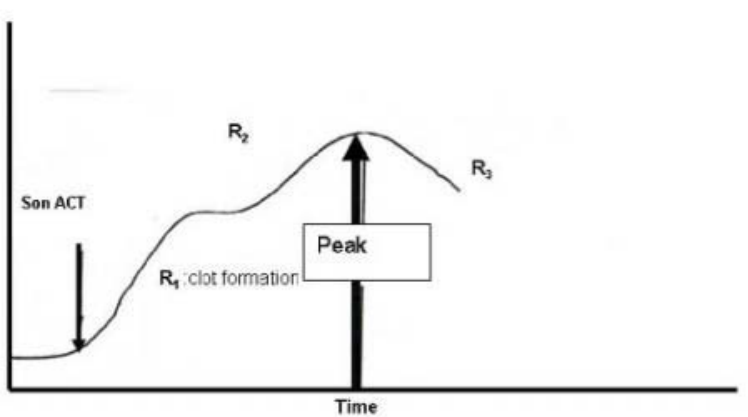

FIGURE 3 Sonoclot variables. Son activated clotting time (ACT) or Sonoclot ACT = onset time from the beginning of the trace until the beginning of fibrin formation. It corresponds to the conventional ACT measurement using the Hemochron ${ }^{\circledR}$ method. $\mathrm{Rl}$ slope $=$ clot rate expressed as a percentage of the peak amplitude per unit time (normal range: $15-45 \%)$. Inflection point $=$ between $\mathrm{Rl}$ and $\mathrm{R} 2$ slopes. It represents the start of contraction of the fibrin strands by the action of platelets. R2 slope = reflects fibrinogenesis, fibrin polymerization and platelet-fibrin interactions. Peak impedance reflects completion of fibrin formation. The amplitude of the peak is linked to fibrinogen concentration. The time to peak amplitude reflects the rate of conversion of fibrinogen to fibrin. R3 slope = downward slope is produced as platelets induce further contraction of the clot. Hypofibrinogenemia manifests itself with a short R2 and small peak amplitude. Thrombocytopenia or poor platelet function result in a prolonged inflection, shallow R2 and R3 slopes and a large residual signal after R3.

Results can be assessed within 30 min by adding celite, an activator of coagulation. A modified TEG device $\left(\right.$ ROTEG $^{\mathrm{TM}}$ system) that allows rapid testing in 15-20 min is presently under evaluation. ${ }^{28}$

Differences have been observed between arterial and venous blood values, a stronger clot formation having been reported for arterial blood samples; gender related differences have also been described. Native-blood TEG seems to be more reliable than TEG using recalcified citrated blood. ${ }^{23}$

Thrombelastography is widely used to monitor coagulation and clot lysis in cardiac surgery, liver transplantation, trauma and obstetrics. It may be useful to predict bleeding, however, results are contradictory. For some authors, the maximal amplitude might be more predictive than routine tests after cardiac surgery to predict postoperative bleeding. For others, conventional tests have a better sensitivity and specificity to predict bleeding. The TEG has also been used in algorithms to determine the indications for blood transfusion in liver transplantation and cardiac 
surgery. Despite its widespread use in clinical practice, the TEG has not been validated and has never been standardized. As for the tests described previously, collaborative studies with hematologists will be necessary to determine the clinical usefulness of this POC monitor of coagulation.

The Sonoclot (SCT; Sienco Inc., Morrison, CO, USA) device uses a small probe driven by ultrasonic vibration and measures the impedance of clotting upon the vibratory pattern. As for the TEG, cuvettes are available with various types of activators to accelerate coagulation and to measure specific variables. The instrument has been used to evaluate platelet function and defects in coagulation factors during liver transplantation and after cardiac surgery. It may be predictive of bleeding after cardiopulmonary bypass. Unlike the TEG, the SCT has not been used to guide transfusion schemes. ${ }^{29,30}$ In clinical practice, measurements are less reproducible with the SCT than with the TEG. ${ }^{31}$

In conclusion, the laboratory investigation of hemostasis should be oriented by clinical examination and circumstances. Preoperative laboratory tests should not be prescribed systematically. When needed, this investigation proceeds by successive steps from screening tests to more specific and complex assays. Preanalytical variables are of paramount importance for the quality of the results. Point of care systems, which avoid delays in sample transmission, address various aspects of hemostasis, from primary hemostasis to global coagulation and fibrinolysis. Although the analytical performances of these systems are excellent, their advantage over the conventional laboratory approach has rarely been documented by large studies of high methodological quality. Consequently, the benefit of their use, in terms of quality of care or costutility, remains difficult to extrapolate to the general practice.

\section{References}

1 Houry S, Georgeac C, Hay JM, Fingerhut A, Boudet MJ. A prospective multicenter evaluation of preoperative hemostatic screening tests. The French Associations for Surgical Research. Am J Surg 1995; 170: 19-23.

2 Burk CD, Miller L, Handler SD, Cohen AR. Preoperative history and coagulation screening in children undergoing tonsillectomy. Pediatrics 1992; 89: 691-5.

3 Janvier G. Preoperative evaluation of hemorrhagic risk. Ann Fr Anesth Reanim 1998; 17(Suppl 1): 2s-5.

4 Polack B, Schved JF, Boneu B; Groupe d'Etude sur l'Hémostase et la Thrombose (GEHT). Preanalytical recommendations of the 'Groupe d'Etude sur
l'Hémostase et la Thrombose' (GEHT) for venous blood testing in hemostasis laboratories. Haemostasis 2001; 31: 61-8.

5 Veyradier A, Fressinaud E, Meyer D. Laboratory diagnosis of von Willebrand disease. Int J Clin Lab Res 1998; 2: 201-10.

6 Buchanan MR, Brister SJ. Individual variation in the effects of ASA on platelet function: implications for the use of ASA clinically. Can J Cardiol 1995; 11: 221-7.

7 Peterson P, Hayes TE, Arkin CF, et al. The preoperative bleeding time test lacks clinical benefit: College of American Pathologists' and American Society of Clinical Pathologists' position article. Arch Surg 1998; 133: 134-9.

8 Gewirtz AS, Miller ML, Keys TF. The clinical usefulness of the preoperative bleeding time. Arch Pathol Lab Med 1996; 120: 353-6.

9 De Caterina R, Lanza M, Manca G, Strata GB, Maffei $S$, Salvatore L. Bleeding time and bleeding: an analysis of the relationship of the bleeding time test with parameters of surgical bleeding. Blood 1994; 84: 3363-70.

10 Jilma B. Platelet function analyzer (PFA-100): a tool to quantify congenital or acquired platelet dysfunction. J Lab Clin Med 2001; 138: 152-63.

11 Fressinaud E, Veyradier A, Truchand F, et al. Screening for von Willebrand disease with a new analyzer using high shear stress: a study of 60 cases. Blood 1998; 91: 1325-31.

12 Thiagarajan $P, W u K K$. In vitro assays for evaluation platelet function. In: Grisele P, Page CP, Fuster V, Vermylen J (Eds). Platelets in Thrombotic and NonThrombotic Disorders. Cambridge; 2002: 459-70.

13 Arnout J. Antiphospholipid syndrome: diagnostic aspects of lupus anticoagulants. Thromb Haemost 2001; 86: 83-91.

14 Taylor FB Jr, Toh CH, Hoots WK, Wada H, Levi M; Scientific Subcommittee on Disseminated Intravascular Coagulation (DIC) of the International Society on Thrombosis and Haemostasis (ISTH). Towards definition, clinical and laboratory criteria, and a scoring system for disseminated intravascular coagulation. Thromb Haemost 2001; 86: 1327-30.

15 Boneu B, de Moerloose P. How and when to monitor a patient treated with low molecular weight heparin. Semin Thromb Hemost 2001; 27: 519-22.

16 Rapaport SI. Preoperative hemostatic evaluation: which tests, if any? Blood 1983; 61: 229-31.

17 Shore-Lesserson L. Point-of-care coagulation monitoring for cardiovascular patients: past and present. J Cardiothorac Vasc Anesth 2002; 16: 99-106.

18 Despotis GJ, Levine V, Saleem R, Spitznagel E, Joist JH. Use of point-of-care test in identification of patients who can benefit from desmopressin during cardiac sur- 
gery: a randomised controlled trial. Lancet 1999; 354: 106-10.

19 Isgro F, Rehn E, Kiessling AH, Kretz KU, Kilian W, Saggan $W$. Platelet function test HemoSTATUS 2: tool or toy for an optimized management of hemostasis. Perfusion 2002; 17: 27-31.

20 Forestier F, Coiffic A, Mouton C, Ekouevi D, Chene G, Janvier $G$. Platelet function point-of-care tests in postbypass cardiac surgery: are they relevant? $\mathrm{Br} \mathrm{J}$ Anaesth 2002; 89: 715-21.

21 Despotis GJ, Levine V, Filos KS, et al. Evaluation of a new point-of-care test that measures PAF-mediated acceleration of coagulation in cardiac surgical patients. Anesthesiology 1996; 85: 1311-23.

22 Fattorutto M, Pradier O, Schmartz D, Ickx B, Barvais $L$. Does the platelet function analyser (PFA-100) predict blood loss after cardiopulmonary bypass? Br J Anaesth 2003; 90: 692-3.

23 Samama CM, Ozier $\Upsilon$. Near-patient testing of haemostasis in the operating theatre: an approach to appropriate use of blood in surgery. Vox Sang 2003; 84: 251-5.

24 Havrda DE, Hawk TL, Marvin CM. Accuracy and precision of the CoaguChek $S$ versus laboratory INRs in a clinic. Ann Pharmacother 2002; 36: 769-75.

25 Kemme MJ, Faaij RA, Schoemaker RC, et al. Disagreement between bedside and laboratory activated partial thromboplastin time and international normalized ratio for various anticoagulants. Blood Coagul Fibrinolysis 2001; 12: 583-91.

26 Despotis GJ, Santoro SA, Spitznagel E, et al. On site prothrombin time, activated partial thromboplastin time, and platelet count. A comparison between whole blood and laboratory assays with coagulation factor analysis in patients presenting for cardiac surgery. Anesthesiology 1994; 80: 338-51.

27 Mallett V, Cox DJ. Thrombelastography. Br J Anaesth 1992; 69: 307-13.

28 Cammerer U, Dietrich W, Rampf T, Braun SL, Richter $J A$. The predictive value of modified computerized thromboelastography and platelet function analysis for postoperative blood loss in routine cardiac surgery. Anesth Analg 2003; 96: 51-7.

29 Hett DA, Walker D, Pilkington SN, Smith DC. Sonoclot analysis. Br J Anaesth 1995; 75: 771-6.

30 Nijhawan N, Spiess BD. The intraoperative evaluation of haemostasis. Anesth Clin North America 1999; 17: 733-47.

31 Forestier F, Belisle S, Constant C, Harel F, Janvier G, Hardy JF. Reproductibility and interchangeability of the Thromoelastograph, Sonoclot and Hemochron activated coagulation time in cardiac surgery. Can J Anesth 2001; 48: 902-10. 